\title{
Diagnóstico precoz y prevención de la enfermedad ateroesclerótica sistémica
}

\author{
Óscar Román
}

Departamento de Medicina Campus Central. Facultad de Medicina. Universidad de Chile.

\section{Resumen:}

Existe evidencia significativa en varias comunicaciones que los sujetos con presión arterial ligeramente sobre el nivel normal de 120/80 mmHg, pero bajo 140/90 mmHg, considerados pre-hipertensos en los últimos Consensos internacionales, presentan signos precoces de alteración cardiovascular propios de la enfermedad arterioesclerótica. Además, estos cambios iniciales se presentan asociados a algunos factores de riesgo $\mathrm{C}-\mathrm{V}$, en especial de diabetes, dislipidemia, obesidad y sedentarismo. Por tanto, se ha propuesto cambiar el paradigma basado en las cifras de PA por otro que involucre las alteraciones precoces y los factores de riesgo. En esta etapa inicial de la enfermedad arterial, es preciso evaluar su riesgo multifactorial a 5 o 10 años plazo, y de acuerdo a éste, planificar las medidas terapéuticas de manejo de los FR y del posible uso de drogas antihipertensivas. De este modo se podrá implementar una profilaxis primaria racional y muy posiblemente eficaz.

Palabras clave: pre-hipertensión Daño C-V precoz. Prevención primaria.

\section{Introducción:}

Estimar el valor de la innovación en Medicina es un desafío continuo ${ }^{1}$. A pesar de evidencias de nuevos conceptos y enfoques sobre algunas enfermedades, sustentados por ensayos clínicos confiables, la modificación de definiciones y paradigmas y por tanto, su impacto sobre la práctica médica y sobre los consensos aceptados en la literatura es lento y difícil. Un ejemplo de esta situación lo constituye la Hipertensión arterial (HTA), que ha sido considerada clásicamente como una enfermedad específica ${ }^{2}$. Sin embargo, desde los estudios fundacionales en Framingham de la teoría de los factores de riesgo $(\mathrm{FR})^{3}$, la hipertensión arterial primaria o esencial ha sido considerada también como un factor de riesgo más de la enfermedad ateroesclerótica sistémica, al igual que lo han sido la diabetes y la dislipidemia ${ }^{4,5}$.

\section{Correspondencia:}

Dr. Oscar Román A

Huelén 154 Dto. 2 Prov.

Santiago.

Email:oscarromanalemany@hotmail.com
Desde esa época, la denominada Enfermedad hipertensiva, considerada una entidad clínico patológica definida, en los sucesivos estudios internacionales multicéntricos controlados, mostró que presentaba los mismo factores de riesgo de la Enfermedad coronaria y de otras patologías ateroescleróticas ${ }^{6,7,8}$. Además sus complicaciones, letales o no, eran exactamente las mismas que las de la enfermedad ateroesclerótica sistémica ${ }^{5,9,10}$.

De acuerdo a estas observaciones, desde hace más de una década, se postuló un nuevo concepto: “considerar a la hipertensión arterial sólo un marcador de un proceso degenerativo y proliferativo cardiovascular que desemboca en las lesiones ateroescleróticas clásicas ${ }^{11,12,13,14}$.

Este nuevo paradigma, en líneas generales, está fundamentado en una serie de hallazgos epidemiológicos, 
clínicos, fisiopatológicos y terapéuticos, que describiremos a continuación.

A). Antecedentes Epidemiológicos. Se ha podido demostrar que la HTA se desarrolla paralelamente a algunos factores de riesgo conocidos de la enfermedad ateroesclerótica, como la diabetes, la hipercolesterolemia y la obesidad. Además, los cambios de uno u otro factor son coincidentes con gran frecuencia y en similar magnitud ${ }^{15}$.

Los FR conocidos, tanto constituyentes de estilo de vida como determinados por cambios fisiopatológicos precoces (hipercolesterolemia, hiperglicemia), se asocian a un mayor riesgo de desarrollar enfermedad coronaria y ateroesclerosis de otros órganos ${ }^{16}$. De éstos, los más importantes son diabetes, tabaquismo, dislipidemia, obesidad y sedentarismo $2,3,5,8$.

Hasta el año 2003, en que se comunicó el $7^{\circ}$ Consenso 8 , el riesgo de eventos C-V había sido estudiado epidemiológicamente en función de cada FR identificado. La reducción por separado de cada uno de ellos, específicamente de los modificables, permitía una disminución de los eventos, estableciéndose así una base de prevención primaria ${ }^{1,9,17}$. Como esos estudios demostraron que la HTA era un FR más de la ateroesclerosis, se llegó a postular que ella no era una enfermedad aislada y específica, sino un factor de riesgo más, se ha llegado a postular que la HTA no sería una enfermedad aislada y específica, sino un factor de riesgo más de la enfermedad ateroesclerótica, aunque muy importante y significativo ${ }^{13,14}$.

Si bien hasta el Consenso $7^{\circ}$ se había reconocido el rol por separado de los FR conocidos, desde esa época y aún antes se había estudiado el riesgo total, considerando a todos los factores de riesgo comprometidos en cada paciente. Se estableció así un criterio multifactorial, o sea, que el riesgo absoluto de desarrollar eventos C-V sería función de la interacción de todos los FR identificados y no de uno solo ${ }^{9,15,16,17}$.

En apoyo de esta concepción, se ha observado que hipertensión, glucosa y colesterol se distribuyen en forma continua en la población, de manera que cualquier aumento de los niveles de uno de ellos a través de sus respectivos rangos, aumenta el riesgo absoluto de de desarrollar eventos C-V. Por tanto, la definición de rangos de normalidad de hipertensión, dislipidemia y diabetes es arbitraria. Ello, porque la hipertensión se ha definido como el nivel de PA sobre el cual los estudios randomizados revelan que reduciéndolo, disminuye el riesgo $\mathrm{C}-\mathrm{V}$. Para la diabetes, ésta se define cuando el nivel de glicemia se asocia con un aumento del riesgo de determinar las complicaciones microvasculares, en particular, la retinopatía. ${ }^{15}$.

Por lo anterior, debe reconocerse que individuos con presiones inferiores a los niveles de normalidad tienen todavía riesgo de desarrollar enfermedad C-V, y lo mismo es válido para el colesterol y la glucosa. Así se ha postulado un nuevo paradigma basado en el concepto de un riesgo $\mathrm{C}-\mathrm{V}$ total o absoluto, en el cual se deben precisar las definiciones terapéuticas ${ }^{19}$. Este nuevo concepto establece que si la etiología de la Enfermedad C-V es multifactorial, el riesgo total de desarrollarla es función integral de todos esos factores, siendo la hipertensión sólo una parte. Así, por ejemplo, un individuo con PA sistólica de $154 \mathrm{mmHg}$, que no ha fumado en toda su vida y con colesterol normal, tiene un riesgo total menor comparado con otro que tiene PAS más baja (144 mmHG) pero con tabaquismo crónico y colesterol elevado. Por ello, para tratar un determinado FR es necesario tomar en cuenta la contribución colectiva de los otros factores. Con este propósito se han construido tablas de riesgo multifactorial para manejar las recomendaciones terapéuticas, tales como las de Nueva Zelandia y de las Sociedades Europeas de Hipertensión, Cardiología y Ateroesclerosis ${ }^{18,19}$.

2.- Antecedentes Clínicos. Las ventajas de una aproximación epidemiológica respecto a los FR son varias. Por ejemplo, clínicamente se puede asegurar falsamente a los pacientes que sus FR son todos normales, pero el riesgo multifactorial puede estar elevado. Además, en base al riesgo total se le puede indicar a los pacientes cambio en los estilos de vida y considerar el uso de drogas cuando sea necesario. Por tanto el nuevo paradigma permite informar al paciente de su verdadero riesgo total y no catalogarlo como hipertenso o asegurarle falsamente que su PA es normal.

La Tablas europeas informan un riesgo $\mathrm{C}-\mathrm{V}$ total $>$ $5 \%$ de enfermedad fatal a 10 años como umbral en el cual debe considerarse el tratamiento. Estas tablas permiten, además, evitar tratamientos innecesarios en sujetos con elevación de un solo FR pero con riesgo multifactorial bajo.

La desventaja de esta estrategia es que concentra el tratamiento en las personas de edad avanzada, porque el riesgo aumenta con la edad, lo que refleja en el fondo una vida larga expuesta a FR y malos estilos de vida $^{15}$.

Cuando las Tablas y guías terapéuticas se convierten en indicadores prioritarios del manejo clínico, obligan a mirar al futuro en cuanto al desarrollo de eventos finales. Pero para hacer prevención, se hace indispensa- 
ble buscar indicadores del curso del proceso $\mathrm{C}-\mathrm{V}$ en ciernes o en etapas iniciales. Si se usan indicadores finales como el daño de órganos blanco (infarto miocárdico, AVE, insuficiencia renal, cardíaca, etc.), puede suceder que las diferencias biológicas y de conducta de los personas hayan sido mucho más determinantes del pronóstico que el tratamiento mismo ${ }^{20}$.

3.- Antecedentes fisiopatológicos. Por las consideraciones anteriores, algunos autores han postulado que las mejores guías terapéuticas de la enfermedad $\mathrm{C}-\mathrm{V}$ desde sus etapas iniciales, son los marcadores de daño, en conjunto con los FR. Algunos investigadores apuntan a que el manejo clínico no debe basarse exclusivamente en las cifras de PA o en los niveles de otros FR como indicadores fieles ${ }^{12,14,21}$. Sin embargo, clásicamente se ha reconocido que el riesgo de morbimortalidad C-V sigue una correlación lineal directa con los niveles de la presión arterial, siendo mayor en las hipertensiones severas. No obstante, algunos estudios han comprobado que el riesgo se mantiene, aunque bajo, con niveles de PA considerados normales, esto es $<$ de 140/90 mmHg, ${ }^{22,23}$. Ello llevó a considerar niveles de pre-hipertensión y aún de hipertensión Etapa 1 , a los comprendidos entre $121 / 139$ y $81 / 89$ para la sistólica y diastólica, respectivamente. En este rango se han observado signos precoces de daño estructural y funcional del corazón y de los vasos ${ }^{14,23}$. Por esta razón se ha llamado la atención, además de los FR conocidos, a otros marcadores de riesgo que pueden influir en la prevención y tratamiento de la hipertensión en función de evitar los eventos mayores de daño C-V (IAM, AVE, etc).

En base a estos antecedentes, se ha postulado un cambio de paradigma para establecer el riesgo $\mathrm{C}-\mathrm{V}$, deri- vado de la HTA, considerando que a largo plazo, ella evoluciona desde niveles de PA discretos que pueden determinar daños estructurales presentes en todos los órganos del sistema C-V y que en la evolución a largo plazo, pueden condicionar el pronóstico en cuanto a eventos letales y no letales conocidos.

Los marcadores de daño precoz $\mathrm{C}-\mathrm{V}$ son diversos y localizados en distintos órganos. Se los puede distinguir en anatomopatológicos y fisiopatológicos. En la Tabla 1 se anotan los más significativos y de utilidad en la práctica clínica.

Estos marcadores pueden estar ya presentes en la etapa denominada pro-hipertensión, o sea entre cifras de $129 / 80$ y 139/89 mmHg., por lo que esta etapa, que sigue a la normalidad, se le designa como Etapa 1 de Hipertensión por algunos autores ${ }^{14}$. Esta también acepta la presencia de escasos factores de riesgo y aún su ausencia.

4.-Antecedentes terapéuticos.- Respecto a la hipótesis que en esta etapa la intervención terapéutica con drogas confiera protección y mejoría del pronóstico en cuanto a lesiones estructurales, aún no hay estudios randomizados que la apoyen fuertemente. Pero es preciso reconocer que a la larga, pueden desarrollar un alto riesgo de desarrollar cifras de PA sobre 140/90 $\mathrm{mmHg}$. Por tanto, en forma preventiva, es necesario precisar el riesgo multifactorial a 5 años con las Tablas de Framingham ${ }^{24}$.

Actualmente se recomienda en esta población prehipertensiva, determinar la existencia de los factores de riesgo incluidos en las Tablas ya conocidas, inicia un tratamiento integral y cooperativo de todos esos factores, y en las personas con diabetes y enfermedad renal es recomendable iniciar terapia con drogas antihiper-

\begin{tabular}{|c|c|}
\hline Sistema & Alteración fisiopatológica precoz. \\
\hline Presi $\mathrm{n}$ arterial & $\begin{array}{l}\text { P rdida del Dip nocturno } \\
\text { Respuesta exagerada de PA al ejercicio. } \\
\text { Aumento de la Presi } n \text { de pulso }\end{array}$ \\
\hline Corazón & $\begin{array}{l}\text { Discreta hipertrofia ventricular izquierda } \\
\text { Aumento de la presión de llena de aurícula izqda.. }\end{array}$ \\
\hline Arterias & $\begin{array}{l}\text { Rigidez de arterias centrales } \mathrm{y} \text { arteriolas. } \\
\text { Aumento del espesor intima-media car tidas } \\
\text { Disfunci } \mathrm{n} \text { endotelial }\end{array}$ \\
\hline Ri $n$ & $\begin{array}{l}\text { Microalbuminuria } 30 \text { a } 300 \mathrm{mg} / \mathrm{d} \\
\text { Creatininemia elevada }\end{array}$ \\
\hline Retina & Espasmo y engrosamiento arterias \\
\hline
\end{tabular}


tensivas 14. Sin embargo, es necesario esperar los resultados de nuevos ensayos multicéntrico en los hipertensos Etapa 1 para establecer si el tratamiento para reducir la PA podría detener o demorar la progresión de la enfermedad ateroesclerótica.

En la mirada general al tratamiento, es preciso reconocer diversas falencias de éste en el largo plazo. Se han identificado algunos factores que pueden influir en el manejo terapéutico adecuado de los hipertensos, a saber, la no sujeción de los médicos a las indicaciones de los Consensos y el desconocimiento de los pacientes de su condición patrológica. Además influye la adhesividad del paciente a las medidas de control de FR y de las drogas antihipertensiva. Por otro lado, en algunos de estos aspectos intervienen además el nivel cultural y socioeconómico de los sujetos ${ }^{20}$.
Desde el punto de vista de la prevención, se han comunicado dos extensos estudios multicéntricos europeos en prevención secundaria de enfermos coronarios, EUROSPIRE I y II ${ }^{25.26}$, que demostraron que la meta de 140/90 mmHg no se alcanzó en el 50\% de los pacientes, a pesar del uso liberal de fármacos y del control de los FR, y que además, un $25 \%$ de los pacientes al inicio, desconocían su condición de hipertensos.

En cuanto a prevención primaria, es preciso esperar los resultados de algunas investigaciones que, en pacientes en etapa de pre-hipertensión, traten grandes poblaciones con factores de riesgo como diabetes y enfermedad renal. A la vez, se requieren nuevas investigaciones para establecer la confiabilidad y validez de la nueva clasificación en etapas de la Hipertensión basada en la presencia de marcadores precoces de alteraciones de arterioesclerosis.

\section{Referencias:}

1. Long G, Cutler DM, Berndt ER. Antihypertensive drugs: a perspective on the value of improved blood pressure control in the USA. Europ Heart J 2007; 9: B19-B22.

2. Kaplan NM. Clinical hypertension Sixth Ed. 1994. W \&W. Baltimore. USA.

3. Kannel WB. Importance of hypertension as a mayor risk factor in cardiovascular disease.In "Hypertension”, 1977 Mc Graw Hill, pags $88-910$

4. Pooling Project Research Group. Relationship of blood pressure, serum cholesterol, smoking habit, relative weight and ECG abnormalities to incidence of major coronary events: Final report of the Pooling Project Research Group. J Chronic Dis 1975; 31 : 201-306.

5. Multiple risk factor intervention trial. Risk factor changes and mortality results. Multiple Risk Factor Intervention Trial Research Group. JAMA. 1982; 248: 1465-1477.

6. Joint National Committee on Detection, Evaluation, and Treatment of High Blood Pressure. The fifth report of the Joint National Committee on detection, evaluation and treatment of high blood pressure. Arch Intern Med 1993; 153: 154-93.

7. Chobanian AV, Bakris GL, Black HR, Cushman WC, Green LA et al. Seventh report of the Joint National Commitee on Prevention, Detection, Evaluation and Treatment of High Blood Pressure. Hypertension 2003; 42:1206-1252.

8. Roman O,Meza N.Arterial hypertension: end organ damage and its prevention. Intercontinental Cardiology 1996; 5:49-56.

9. Román O, Badilla M, Cuevas G, Valenzuela MA, Barahona S,
Baez D, et al. Morbimortalidad de la hipertensión arterial según magnitud del cambio de los factores de riesgo. Seguimiento a 30 años. Rev Chil Cardiol 2005; 24: 11

10. Glagov S, Zarins C, Giddens DP, Ku DN. Hemodynamics and aterosclerosis: insights and perspectives gained from studies of human arteries. Arch Pathol Lab Med 1988; 112: 1018-31.

11. Julius S, Jamerson K, Mejia A, Krause L, Schork N, Jones K. The association of borderline hypertension with target organ changes and higher coronary risk. Tecumseh Blood Pressure study. JAMA 1990; 264: 354-58.-

12. Roman O. La enfermedad cardiovascular ¿una nueva entidad? Relación de la hipertensión arterial con ella. Bol Soc Ch Hypertens 1998; 7: 3-5.

13. Giles TD, Berk BC, Black HR, Cohn JR, Kostis JB, Izzo JL, et al. Expanding the definition and clasification of Hypertension. J Clin Hypertens 2006; 7: 505-12.

14. Wood D. The concepts of doctor targets base on quality guidelines: focus on blood pressure. European Heart J 2007; 9: B29B36.

15. Holme I, Enger SC, Helgeland A, Hjermann I, Leren P, LundLarsen PG, et al. Risk factors and raised atherosclerotic lesions in coronary and cerebral arteries. Statistical analysis from the Oslo study. Ateroesclerosis 1981; 1: 250-56.

16. Kannel W. Blood pressure as a cardiovascular risk factor prevention and treatment. JAMA 1996; 275:1571-76.

17. Jackson R, Barham P, Bills J, Birch T, Macmahon S, Maling T. Management of raised blood pressure in New Zealand: a discussion document. Br Med J 1993; 307: 107-110.

18. De Backer G, Ambrosioni E, Borch-Johnsen K, Brotons C, Cifkova R, Dallongeville J, et al. European guidelines on cardio- 
Diagn stico precoz y prevenci $n$ de la enfermedad ateroescler tica sist mica

Rom n O, et al.

vascular disease prevention in clinical practice. Third Joint Task Force of European and other Societies on Cadiovascular Disease Prevention in Clinical Practice. Eur J Cardiovasc Prevention Rehabil 2003; 10: S1-S10.

19. Califf RM. The cycle of quality as a model for improving health outcomes in the treatmente of hypertension. Eur Heart J 2007; 9: B8-B12.

20. Wang Y, WANG Q H. The prevalence of prehypertension and hypertension among US adults according to the new joint national committee guidelines: new challenges of the old problem. Arch Inter Med 2004; 164: 2126-34.

21. MacMahon S, Peto R, Cutler J, Collins R, Sorlie P, Neaton J, et al. Blood pressure, stroke, and coronary heart disease. Part 1, Prolonged differences in blood pressure: prospective observational studies corrected for the regression dilution bias. Lancet 1990; 335: $765-73$.

22. Vasan RS, Larson MG, Leip EP, Evans JC, O’Donnell CJ, Kannel WB, et al. Impact of high-normal blood pressure on the risk of Cardiovascular disease. N Engl J Med 2001; 345: 1291-97.

23. Grundy SM, Pasternak R, Greenland P, Smith S Jr, Fuster V. AHA/ACC scientific statement: Assessment of cardiovascular risk by use of multiple-risk-factor assessment equations: a statement for healthcare professionals from the American Heart Association and the American College of Cardiology. J Am Coll Cardiol 1999; 34: 1348-59.

24. Lifestyle and risk factor management and use of drug therapies in coronary patients from 15 countries; principal results from EUROASPIRE II Euro Heart Survey Programme. Eur Heart J. 2001; 22: 554-572.

25. Clinical reality of coronary prevention guidelines: a comparison of EUROASPIRE I and II in nine countries. Lancet 2001; 357: 995-1001.

Hypertens 2006; 7: 505-12.

14. Wood D. The concepts of doctor targets base on quality guidelines: focus on blood pressure. European Heart J 2007; 9: B29B36.

15. Holme I, Enger SC, Helgeland A, Hjermann I, Leren P, LundLarsen PG, et al. Risk factors and raised atherosclerotic lesions in coronary and cerebral arteries. Statistical analysis from the Oslo study. Ateroesclerosis 1981; 1: 250-56.
16. Kannel W. Blood pressure as a cardiovascular risk factor prevention and treatment. JAMA 1996; 275:1571-76.

17. Jackson R, Barham P, Bills J, Birch T, Macmahon S, Maling T. Management of raised blood pressure in New Zealand: a discussion document. Br Med J 1993; 307: 107-110.

18. De Backer G, Ambrosioni E, Borch-Johnsen K, Brotons C, Cifkova R, Dallongeville J, et al. European guidelines on cardiovascular disease prevention in clinical practice. Third Joint Task Force of European and other Societies on Cadiovascular Disease Prevention in Clinical Practice. Eur J Cardiovasc Prevention Rehabil 2003; 10: S1-S10.

19. Califf RM. The cycle of quality as a model for improving health outcomes in the treatmente of hypertension. Eur Heart J 2007; 9 : B8-B12.

20. Wang Y, WANG Q H. The prevalence of prehypertension and hypertension among US adults according to the new joint national committee guidelines: new challenges of the old problem. Arch Inter Med 2004; 164: 2126-34.

21. MacMahon S, Peto R, Cutler J, Collins R, Sorlie P, Neaton J, et al. Blood pressure, stroke, and coronary heart disease. Part 1, Prolonged differences in blood pressure: prospective observational studies corrected for the regression dilution bias. Lancet 1990; 335: 765-73.

22. Vasan RS, Larson MG, Leip EP, Evans JC, O’Donnell CJ, Kannel WB, et al. Impact of high-normal blood pressure on the risk of Cardiovascular disease. N Engl J Med 2001; 345: 1291-97.

23. Grundy SM, Pasternak R, Greenland P, Smith S Jr, Fuster V. AHA/ACC scientific statement: Assessment of cardiovascular risk by use of multiple-risk-factor assessment equations: a statement for healthcare professionals from the American Heart Association and the American College of Cardiology. J Am Coll Cardiol 1999; 34: 1348-59.

24. Lifestyle and risk factor management and use of drug therapies in coronary patients from 15 countries; principal results from EUROASPIRE II Euro Heart Survey Programme. Eur Heart J. 2001; $22:$ 554-572.

25. Clinical reality of coronary prevention guidelines: a comparison of EUROASPIRE I and II in nine countries. Lancet 2001; 357 : 995-1001. 